\title{
[gw22-e1025] PRACTICE STUDY ON MASSAGE EFFECTS ON STRESS RESPONSE OF PATIENTS AWAITING CARDIOVASCULAR SURGERY
}

Yang Chun-yan Shanxi Medical University School of Nursing, Shanxi, China

10.1136/heartjnl-2011-300867.500

Objective To investigate the trait-state anxiety, pain and efficacy of massage in patients awaiting Cardiovascular surgery and to assess overall patient satisfaction with massage. To observe the stress objective index before and in the operation, and then evaluate the feasibility of integrating massage into preoperative practices.

Methods Select 50 patients who will experience cardiovascular surgery in the second of Shanxi medical university from October 2010 to December 2010, and allocate them randomly to two groups: control group $(n=26)$ and intervention group $(n=24)$. The control group patients received standard preoperative care, and intervention group received massage care.

Results The massage group intervention was more effective than the standard care group for alleviating pain degree $(p<0.006)$, muscle state-anxiety $(\mathrm{p}<0.002)$. The intervention groups' pain degree $(2.29 \pm 1.23)$ during operation was significantly lower than the control group (4.46 \pm 1.92$)$. The $95 \%$ patients of the intervention group thought the nursing interventions helpful.

Conclusion Patients' operation stress reaction reflected in anxiety, heart rate, systolic and diastolic blood pressure, pain degree. To compare the data, the results manifest the nursing interventions (massage) do help for stress relief, degrade preoperative anxiety and stress reaction during operation, raise the tolerance ability to pain, and help patients successfully live through operation and inchoate recovery. 